\title{
3D printing and intraoperative neuronavigation tailoring for skull base reconstruction after extended endoscopic endonasal surgery: proof of concept
}

\author{
Walid I. Essayed, M.D. ${ }^{*}$, , Prashin Unadkat, M.D. ${ }^{2}$, Ahmed Hosny, M.S. ${ }^{3}$, Sarah Frisken, \\ Ph.D. ${ }^{2}$, Marcio S. Rassi, M.D. ${ }^{1}$, Srinivasan Mukundan Jr., M.D. Ph.D. ${ }^{2}$, James C. Weaver, \\ Ph.D. ${ }^{4}$, Ossama Al-Mefty, M.D. ${ }^{1}$, Alexandra J. Golby, M.D. ${ }^{1,2}$, and lan F. Dunn, M.D. ${ }^{1}$ \\ ${ }^{1}$ Department of Neurosurgery, Brigham and Women's Hospital, Harvard Medical School, Boston, \\ MA, USA \\ 2Department of Radiology, Brigham and Women's Hospital, Harvard Medical School, Boston, MA, \\ USA \\ ${ }^{3}$ Department of Radiation Oncology, Dana-Farber Cancer Institute, Brigham and Women's \\ Hospital, Harvard Medical School, Boston, MA, USA \\ ${ }^{4}$ Wyss Institute for Biologically Inspired Engineering, Harvard University, Cambridge, MA, USA
}

\begin{abstract}
Objective-Endoscopic endonasal approaches are increasingly performed for the surgical treatment of multiple skull base pathologies. Preventing postoperative CSF leaks remains a major challenge, particularly in extended approaches. In this study, the authors assessed the potential use of modern multimaterial 3D printing and neuronavigation to help model these extended defects and develop specifically tailored prostheses for reconstructive purposes.
\end{abstract}

Methods-Extended endoscopic endonasal skull base approaches were performed on 3 human cadaveric heads. Preprocedure and intraprocedure CT scans were completed and were used to segment and design extended and tailored skull base models. Multimaterial models with different core/edge interfaces were 3D printed for implantation trials. A novel application of intraoperative landmark acquisition method was used to transfer the navigation, helping to tailor the extended models.

Results-Prostheses were created based on preoperative and intraoperative CT scans. The navigation transfer offered sufficiently accurate data to tailor the preprinted extended skull base

\footnotetext{
*Correspondence: Walid Ibn Essayed, M.D., Department of Neurosurgery, Brigham and Women's Hospital, Harvard Medical School, 75 Francis street, BTM $4^{\text {th }}$ floor, Boston, MA, USA. Phone: 857-307-2312, Fax: 617-713-3050, wibnessayed@bwh.harvard.edu. Author Contributions

Conception and design: Essayed, Unadkat, Hosny, Frisken. Acquisition of data: Essayed, Unadkat, Rassi. Analysis and interpretation of data: Essayed, Unadkat. Drafting the article: Essayed, Hosny. Critically revising the article: Weaver, Golby, Dunn. Reviewed submitted version of manuscript: Golby, Dunn. Approved the final version of the manuscript on behalf of all authors: Essayed. Administrative/technical/material support: Hosny, Frisken, Rassi, Mukundan, Weaver. Study supervision: Mukundan, Al-Mefty, Golby, Dunn.

Disclosures

The authors report no conflict of interest concerning the materials or methods used in this study or the findings specified in this paper.
} 
defect prostheses. Successful implantation of the skull base prostheses was achieved in all specimens. The progressive flexibility gradient of the models' edges offered the best compromise for easy intranasal maneuverability, anchoring, and structural stability. Prostheses printed based on intraprocedure CT scans were accurate in shape but slightly undersized.

Conclusion-Preoperative 3D printing of patient-specific skull base models is achievable for extended endoscopic endonasal surgery. The careful spatial modeling and the use of a flexibility gradient in the design helped achieve the most stable reconstruction. Neuronavigation can help tailor preprinted prostheses.

\section{Keywords}

Endoscopic; Extended endonasal surgery; Neuronavigation; Printing; Three-Dimensional; Prostheses and Implants; Reconstructive Surgical Procedures; Skull base reconstruction

IN recent years, endoscopic endonasal approaches have become a key option for the surgical treatment of multiple skull base pathologies. ${ }^{10,12,27,43}$ As they developed, these approaches extended beyond the sella to the anterior and posterior skull base compartments through progressively more complex corridors requiring larger areas of bone removal. The quality of the skull base reconstruction of these defects is crucial to avoid postoperative CSF leaks and associated meningitis, representing one of the major challenges for endoscopic skull base surgery. ${ }^{20}$ Since the introduction of the nasoseptal flap, progressive advances in reconstructive methods have helped decrease postoperative CSF leak rates, mainly based on multilayer reconstructive techniques. ${ }^{9,11,36,38}$ Nevertheless, postoperative CSF leaks rates are still relatively high in extended approaches, with the incidence ranging between $5 \%$ to $22 \%$ in experienced centers. ${ }^{16,22,25,41}$ One of the major challenges during the reconstruction is the specific spatial conformation of the skull base defect, particularly when the defect extends over 2 different compartments of the skull base. ${ }^{16}$ The uneven edges of such defects limit the value of classically effective strategies based on flat rigid implants, such as the gasket seal and its modifications. ${ }^{16}$ Some authors have proposed a "button-like" technique, where 2 sheets of facia lata graft are sewn together and then used to fill the defect. ${ }^{29}$ This approach is promising but may be limited by the lack of rigidity of the fascia lata graft. Given this ongoing challenge, in this laboratory investigation, we assessed the feasibility of designing and tailoring prostheses for repairing skull base defects using multimaterial 3D printing and neuronavigation, based on pre- and intraoperative scans.

\section{Methods}

\section{Scanning and Approaches}

Three silicone-injected cadaveric specimens were used for this study. Preoperative thin-cut volumetric bone-window CT scans were performed, allowing the segmentation of extended models of the skull base (ex-models) (Fig. 1). We then performed a different endoscopic extended endonasal approach in each specimen: a transplanum transsphenoidal approach to the sella/suprasellar region; a transcribriform transplanum approach with partial opening of the sella floor; and a transclival approach extending from the floor of the sella to the inferior third of the clivus. Postprocedure thin-cut CT scans, equivalent to intraoperative imaging, were obtained, allowing us to segment the defect and directly tailor the implant based on the 
intraoperative conditions (T-model) (Fig. 2). In total, for each cadaver, we prepared 2 sets of implants: an extended skull base model (Ex-model) based on preoperative CT scans, and a tailored model (T-model), modeled directly according the defect seen on the intraoperative scan (Fig. 3).

\section{Modeling and 3D Printing}

DICOM image reconstruction and segmentation was performed on 3D Slicer (http:// www.slicer.org/; Harvard University). ${ }^{14}$ To ensure implant stability while allowing maneuverability through tight nasal cavities, the model was conceived to have a rigid core and flexible edges. With regard to the center/edge material interface, we explored both abrupt and gradual material transitions (Fig. 3). Postprocessing was carried out on segmented meshes (.stl formats) of both Ex- and T-models to ensure consistent thickness, vary material properties, and add in positioning handles. Postprocessing was carried out in Rhinoceros 3D (Robert McNeel \& Associates). This involved reconstructing the model as a spherical segment by identifying the best-fit sphere to the segmented mesh (Fig. 4). The segment was then thickened to $1 \mathrm{~mm}$, and the outline of the segment was offset inward by 3 $\mathrm{mm}$, delineating rigid (core) and flexible (edge) regions. Locations of positioning handles were identified within the rigid core region and were placed along the normal to the thickened spherical segment at the insertion point. A 2-part mesh, representing 2 materials with an abrupt transition, was then exported to the printer.

An additional step was required to introduce a functional material gradient between the rigid and flexible regions. Taking advantage of the ability of modern multimaterial 3D printers to create digital materials with intermediate material properties between the 2 source resins, we incorporated a graded material interface between the rigid and flexible phases. ${ }^{5}$ Prototypes were printed on the Connex500 multimaterial 3D printer (Stratasys). At each print layer, flexible and rigid material photopolymers of different colors were simultaneously ejected and eventually UV-cured into a single build. The materials used for the core and the edge are the rigid white VeroWhitePlus (RGD835, Stratasys) and flexible transparent TangoPlus (FLX930, Stratasys) respectively. These two materials represented the extremes along the rigidity scale with Vero family being the stiffest and the Tango family the most flexible, representing an approximately 1000-fold modulus difference-the Vero being approximately $1 \mathrm{GPa}$ (gigapascals) and the Tango approximately $1 \mathrm{MPa}$ (megapascals). ${ }^{5}$

\section{Navigation Transfer, Tailoring, and Implantation}

The T-model prostheses, based on intraoperative CT scans, were directly implanted into the bony defects, while the Ex-models were tailored to the operative defect using neuronavigation transfer data. We used the intraoperative landmarks acquisition method available on a clinically available neuronavigation system (Brainlab) to transfer the registration to the Ex-model (Fig. 5). When designing the models, handle positions were selected near specific reference points easily identifiable during surgery (floor of the sella, sphenoidal sinus septal base, etc.). After registering the cadavers to the preoperative CT scan and completing the nasal portion of the approach, we proceeded to acquire these same reference point landmarks previously planned on preoperative scans as intraoperative landmarks. A minimum of 4 landmarks were selected for each cadaver, according to the 
models' handles. Landmarks were chosen based on the surrounding anatomy in the vicinity of the planned craniotomy. This would vary based on the cadaver's anatomy and the planned approach. Some of the frequently used landmarks in our experiments were the medial opticocarotid recesses, the base of the sphenoidal septum at it's caudal and rostral end, the sphenoid and the floor of the sella. After the skull base defect was created, we acquired multiple points along its edges to trace the extent of the defects on the preoperative CT scan. The next step was performed using the "Restore registration with intraoperative points" feature on the navigation system. We selected as intraoperative landmarks points on the Exmodels corresponding to the previously acquired landmarks on the cadaver's skull base (Fig. 5). Then, we instructed the software to restore the registration using these acquired intraoperative landmarks, which allowed us to transfer the registration from the cadaveric head onto the Ex-model without moving the navigation reference (Fig. 6). We then used the tracing of the operative defect to help tailor the Ex-model. The tailoring of the models was possible with ordinary surgical scissor given the relatively flexible nature of the material edges.

\section{Results}

In all 3 cadaver heads, the T-models, constructed based on the intraoperative CT featuring the created skull base defects, underestimated the size of the defect in 1 or 2 dimensions. Consequently, even though the shape was accurate, the T-models did not provide a stable reconstruction.

The use of intraoperative landmark acquisition for navigation transfer was sufficiently accurate to allow navigation on the Ex-models as if the pointer were still on the skull base (Fig. 6). Even though not perfectly accurate, the navigation transfer offered sufficient precision to help tailor the Ex-models. We oversized the model by $5 \mathrm{~mm}$, given the insight provided by the results of the first experiment with the T-models. Successful and stable Exmodel implantation was possible in all 3 cadavers. The semitransparent feature of the material used for the printing helped visualize the edges of the skull base defect facilitating the subdural insertion of the model under direct visual control of surrounding structures. No major differences were noticeable between model implantations in the anterior, middle, and posterior skull base defects.

From a rigidity perspective, the models with a central rigid core and an abrupt transition to the flexible edges were harder to manipulate; the abrupt change in flexibility led to occasional tearing at the core-edge interfaces. The models with a central rigid core and progressively flexible edges offered the best compromise for implantation, and stability (Fig. 7; Video 1). The flexibility of the models' edges facilitated the introduction of the models through the nostril and maneuvering inside the nasal cavity.

\section{Discussion}

Extended skull base approaches are frequently performed for multiple skull base pathologies such as pituitary adenomas, craniopharyngiomas, chordomas, meningiomas, sinonasal malignancy, and multiple other lesions. 7,15,23,27,28,33,40,43 The constant challenge faced by 
surgeons after resection is achieving the best watertight reconstruction of the skull base defect created at the time of surgery. ${ }^{9,20,24,38}$ The effectiveness of this closure has improved over recent years with the development of multilayer reconstruction techniques overlaid by a nasoseptal flap. ${ }^{8,11,19,36}$ Currently, the most commonly used techniques are multilayer-based approaches such as the "gasket seal." with its different variations, and the "button technique." ${ }^{29}$ For a gasket seal closure the surgeon wedges a plate, such as Medpore (Porex), 8,30 in the skull base defect after overlaying a fascia lata graft over the defect, creating a gasket, sandwiching the fascia between the plate and the skull base defect edges and dura. 10,16,27 This technique has proven effective in reconstructing most skull base defects, but as defects extend across different compartments of the skull base in larger approaches, the probability of all defect edges aligning on the same plane decreases, leading to instability and weak points around the gasket edges. The button technique is a more versatile strategy used mostly when the skull base defect edges prevent the effective placement of a plate. It uses 2 layers of facia lata graft sewn together at their center, with one layer lodged in the intradural space while the second layer is spread over the other side of the bony defect. ${ }^{29}$ The inherent limitation of this technique may be the lack of rigidity of the construct due to the flexibility of the material, which can lead to progressive dislodgement from normal CSF pulsations and perioperative pressure surges. In addition to a multilayer reconstruction, other preventive techniques can be implemented to reduce the chance of postoperative CSF leaks. The preoperative placement of a lumbar drain helps protect the construct from CSF pressure surges, ${ }^{1}$ and the use of intrathecal fluorescein, which can be injected at the time of the lumbar drain placement, may help identify and quantify any leak and facilitates intraoperative inspection of the quality of the construct. ${ }^{4,35}$ With implementation of these methods, the risk of postoperative CSF leak decreased progressively from around more than $50 \%$ in early studies to less than $3 \%$ in some recent reports. However, when focusing on extended approaches this risk is still nonnegligible reported around 5\% and higher, even in experienced centers. ${ }^{16,20,22,25,41}$

In this study, we addressed the 3D variability of the surgical defects as one of the principal reconstruction challenges. The skull base defects can vary based on the tumor location and the bone removal required for access, its relationships to important neurovascular structures, and the specific nasal and bony anatomy of the patient. To our knowledge, the use of 3D printing to reconstruct endoscopic skull base defects has not previously been assessed. Our results support that the multimaterial 3D-printed skull base reconstruction prostheses described here can be successfully modeled, printed, and implanted. We verified that defect modeling can be successfully performed based on preoperative scans. Our results confirmed also that using currently available commercial systems and preselected landmarks on preoperative CTs, it is possible to transfer a sufficiently precise registration that can guide the surgeon in tailoring a preprinted skull base model prosthesis. This method seems to be closer to clinical practice as preoperative 3D printing of calvarial and other bony defects is increasingly used in multiple situations. ${ }^{13,21,26,31,34,37}$

The use of neuronavigation transfer using preoperatively selected landmarks matched to references on the printed models was feasible using currently available clinical neuro navigation software. This novel (off-label) use of intraoperative landmark acquisition opens the possibility of tailoring the preprinted skull base model according to intraoperatively 
collected points corresponding to the defect edges. This would avoid the need for multiple nasal insertions and decrease the risk of mis-cutting the preprinted model. The surgeon can always return to the original patient image registration by going back to the previous registration solution, as long as the navigation reference array (star) remains in its original position.

While 3D printing of a defect based on an intraoperatively acquired CT scan is not currently feasible, we did assess this scenario. Specifically, in endoscopic endonasal surgery, intraoperative $\mathrm{CT}$ can help update the navigation and guide drilling when extended bone removal is necessary. Even though currently time-consuming, the modeling and printing speed might become compressible to an intraoperative time frame. In the future, it might be possible to acquire an early intraoperative CT (traditional or cone-beam CT) once the bony opening is completed, giving sufficient time for modeling, printing, and sterilizing the $3 \mathrm{D}$ model while the surgeon is performing the tumor resection. Another challenge with using this technique would be the issue of sterility. Although biocompatible materials for the purposes of 3D printing are available, sterilization can be challenging. It would necessitate using materials that are resistant to fast-sterilization techniques. ${ }^{42}$ Alternatively, one can print in a sterile environment, which requires greater precautions right from sourcing the raw materials used, to the postprinted handling of implants, thus raising significant concerns with regulatory bodies about the adequate sterility for implantation. ${ }^{3}$

We found that straightforward modeling of the defect based on the normal bony anatomy raises challenges, particularly at the level of the sella. During endoscopic endonasal surgery, when the sella floor is opened, simply recreating its concave shape may allow accumulation of CSF facing the construct and would represent an increased risk of CSF fistula formation. We theorized that the defect should adopt a less concave curvature particularly between the planum, the tuberculum, and the superior edge of the clivus. A small convex curvature will preclude CSF from stagnating without exerting pressure on the intradural structures.

As demonstrated by our experiments, modeling the skull base defect directly on the intraoperative CT can be even more challenging as all our tailored models ended up underestimating the size of the defect. We think that this discrepancy might be due to the accumulation of imprecisions throughout the manufacturing process. The scanning parameters and windowing can falsely decrease the defect edges during the segmentation, and the smoothing algorithm necessary for the modeling might also lose a few crucial millimeters from the model's final size. A systematic addition of $5-10 \mathrm{~mm}$ in both the coronal and particularly sagittal dimension appears to be necessary based on our preliminary results. A better understanding and standardization of the design steps and printing techniques could help limit these inaccuracies.

After modeling the 3D surface of the defect, we added handles on the inferior nasal surface of the implant. These handles served 2 purposes. In accordance to other authors' experiences, having handles helped maneuver the defect inside the nasal cavity. ${ }^{16}$ These handles also served as landmarks for directing the registration transfer. These custom handles should have a low profile so as not to preclude the multilayer reconstruction. 
Rigid prosthetic models of extended defects may be difficult to introduce through the nostril and would be difficult to maneuver inside the nasal cavity, entailing increased risk of mucosal injury. The implantation of a rigid implant could also be hazardous to surrounding structures, such as the optic chiasm superiorly, the carotid arteries laterally, and the basilar artery posteriorly), as the model may need to be slightly oversized to remain contained by the edges of the defect and prevent subsidence. The sagittal dimension is typically the safest, as visual control is obtainable, while in a coronal plane, lodging a rigid model might induce risk to the carotid arteries. The use of a flexible material for the models' edges may lessen the risk of anatomical damage during placement. Partial folding of the model could in theory decrease risk of injuring the nasal mucosa. The flexible edges were particularly helpful during the implantation, while the central rigid core offered the necessary rigidity to hold the model in place. Also, the flexible and smooth nature of these edges could decrease risk of injury to surrounding structures during insertion. The semitransparent feature of the flexible portions of the models was also helpful during the insertion. The rigidity gradient design offered the most stable construct, distributing tension across the model, with the best compromise between flexibility for placement and rigidity for implant stability.

As previously demonstrated, functionally graded interfaces offer significant advantages in that they can reduce or eliminate stress concentrations at the junctions between rigid and flexible phases in 3D-printed constructs. ${ }^{5}$ Although the location and design of the graded interfaces in the models described here were chosen such that they simply followed the external contours of the modeled geometry, future efforts could include an additional finite element simulation step, to create a more robust form factor. In addition to functionally graded interfaces, future implant designs could also incorporate biocompatible scaffold materials to support and accelerate tissue ingrowth or swellable polymers for a more secure and adaptable fit. ${ }^{17,18,39}$ In addition to postoperative defect reconstruction, this $3 \mathrm{D}$ printing technique might also be helpful in noniatrogenic CSF leak repairs and other situations, such as empty sella syndrome, where techniques for remodeling bony anatomy are limited. 2,6,32

\section{Limitations}

This purely anatomical study suffers multiple inherent limitations, the most pertinent being the verification of the quality and stability of the reconstruction after model implantation, which was only inspected endoscopically, as postimplantation CT and MR studies of the cadaveric heads would not yield sufficient precision to fully assess the quality of the reconstruction. Overall, cadaveric evaluation of the effective impermeability of any reconstruction is challenging, and further evaluation in a more physiological setting will be necessary. Also, the use of only 3 cadaveric heads might not fully account for the anatomical variations and possible surgical challenges, and the method will need to be further assessed in a larger number of specimens.

\section{Conclusions}

Extended endoscopic endonasal approaches are increasingly indicated for a broad variety of skull base pathologies, but are associated with a subsequent nonnegligible risk of postoperative CSF leaks. 3D printing of skull base defects represents a realistic option for 
improving the currently used multilayer reconstruction techniques. The possibilities offered by currently available neuronavigation tools and increasing availability of intraoperative CT scanners can guide printing, tailoring, and implanting extended skull base models. The careful spatial modeling and the use of a flexibility gradient in the material properties of these models can represent a major advance in skull base endoscopic reconstruction techniques. Further laboratory investigations and clinical evaluations will be necessary for validating the practicality and efficiency of this technique. Recent and future developments in 3D printing methods, with respect to both quality and speed, will help refine the clinical applicability of this approach.

\section{Supplementary Material}

Refer to Web version on PubMed Central for supplementary material.

\section{Acknowledgments}

We gratefully acknowledge the support provided by the National Institutes of Health (NIH) grants P41-EB015898, 5P41-EB015902-20, R01-NS049251, R25-CA 089017. We would also like to thank Brainlab (Feldkirchen, Germany) for providing the Kick® system to the skull base laboratory during the study.

\section{References}

1. Ahmed OH, Marcus S, Tauber JR, Wang B, Fang Y, Lebowitz RA. Efficacy of perioperative lumbar drainage following endonasal endoscopic cerebrospinal fluid leak repair: a meta-analysis. Otolaryngol Head Neck Surg. 156:52-60.2017; [PubMed: 27677601]

2. Alvarez Berastegui GR, Raza SM, Anand VK, Schwartz TH. Endonasal endoscopic transsphenoidal chiasmapexy using a clival cranial base cranioplasty for visual loss from massive empty sella following macroprolactinoma treatment with bromocriptine: case report. J Neurosurg. 124:10251031.2016; [PubMed: 26339851]

3. Bandyopadhyay A, Bose S, Das S. 3D printing of biomaterials. MRS Bull. 40:108-115.2015;

4. Banu MA, Kim JH, Shin BJ, Woodworth GF, Anand VK, Schwartz TH. Low-dose intrathecal fluorescein and etiology-based graft choice in endoscopic endonasal closure of CSF leaks. Clin Neurol Neurosurg. 116:28-34.2014; [PubMed: 24315752]

5. Bartlett NW, Tolley MT, Overvelde JT, Weaver JC, Mosadegh B, Bertoldi K, et al. A 3D-printed, functionally graded soft robot powered by combustion. Science. 349:161-165.2015; [PubMed: 26160940]

6. Bedrosian JC, Anand VK, Schwartz TH. The endoscopic endonasal approach to repair of iatrogenic and noniatrogenic cerebrospinal fluid leaks and encephaloceles of the anterior cranial fossa. World Neurosurg. 82(6 Suppl):S86-S94.2014; [PubMed: 25496641]

7. Cappabianca P, Alfieri A, Colao A, Ferone D, Lombardi G, de Divitiis E. Endoscopic endonasal transsphenoidal approach: an additional reason in support of surgery in the management of pituitary lesions. Skull Base Surg. 9:109-117.1999; [PubMed: 17171126]

8. Cappabianca P, Cavallo LM, Esposito F, Valente V, De Divitiis E. Sellar repair in endoscopic endonasal transsphenoidal surgery: results of 170 cases. Neurosurgery. 51:1365-1372.2002; [PubMed: 12445341]

9. Cavallo LM, Messina A, Esposito F, de Divitiis O, Dal Fabbro M, de Divitiis E, et al. Skull base reconstruction in the extended endoscopic transsphenoidal approach for suprasellar lesions. J Neurosurg. 107:713-720.2007; [PubMed: 17937213]

10. Dhandapani S, Negm HM, Cohen S, Anand VK, Schwartz TH. Endonasal endoscopic transsphenoidal resection of tuberculum sella meningioma with anterior cerebral artery encasement. Cureus. 7:e311.2015; [PubMed: 26430585] 
11. Eloy JA, Shukla PA, Choudhry OJ, Singh R, Liu JK. Challenges and surgical nuances in reconstruction of large planum sphenoidale tuberculum sellae defects after endoscopic endonasal resection of parasellar skull base tumors. Laryngoscope. 123:1353-1360.2013; [PubMed: 23483459]

12. Essayed WI, Singh H, Lapadula G, Almodovar-Mercado GJ, Anand VK, Schwartz TH. Endoscopic endonasal approach to the ventral brainstem: anatomical feasibility and surgical limitations. J Neurosurg. 127:1139-1146.2017; [PubMed: 28084906]

13. Fahmy MD, Jazayeri HE, Razavi M, Masri R, Tayebi L. Three-dimensional bioprinting materials with potential application in preprosthetic surgery. J Prosthodont. 25:310-318.2016; [PubMed: 26855004]

14. Fedorov A, Beichel R, Kalpathy-Cramer J, Finet J, Fillion-Robin JC, Pujol S, et al. 3D Slicer as an image computing platform for the Quantitative Imaging Network. Magn Reson Imaging. 30:13231341.2012; [PubMed: 22770690]

15. Fraser JF, Nyquist GG, Moore N, Anand VK, Schwartz TH. Endoscopic endonasal transclival resection of chordomas: operative technique, clinical outcome, and review of the literature. $\mathrm{J}$ Neurosurg. 112:1061-1069.2010; [PubMed: 19698043]

16. Garcia-Navarro V, Anand VK, Schwartz TH. Gasket seal closure for extended endonasal endoscopic skull base surgery: efficacy in a large case series. World Neurosurg. 80:563-568.2013; [PubMed: 22120292]

17. Gladman AS, Matsumoto EA, Nuzzo RG, Mahadevan L, Lewis JA. Biomimetic 4D printing. Nat Mater. 15:413-418.2016; [PubMed: 26808461]

18. Guiducci L, Weaver JC, Bréchet YJM, Fratzl P, Dunlop JWC. The geometric design and fabrication of actuating cellular structures. Adv Mater Interfaces. 2:1500011.2015;

19. Hadad G, Bassagasteguy L, Carrau RL, Mataza JC, Kassam A, Snyderman CH, et al. A novel reconstructive technique after endoscopic expanded endonasal approaches: vascular pedicle nasoseptal flap. Laryngoscope. 116:1882-1886.2006; [PubMed: 17003708]

20. Harvey RJ, Parmar P, Sacks R, Zanation AM. Endoscopic skull base reconstruction of large dural defects: a systematic review of published evidence. Laryngoscope. 122:452-459.2012; [PubMed: 22253060]

21. Hung BP, Naved BA, Nyberg EL, Dias M, Holmes CA, Elisseeff JH, et al. Three-dimensional printing of bone extracellular matrix for craniofacial regeneration. ACS Biomater Sci Eng. 2:1806-1816.2016; [PubMed: 27942578]

22. Jeswani S, Nuño M, Wu A, Bonert V, Carmichael JD, Black KL, et al. Comparative analysis of outcomes following craniotomy and expanded endoscopic endonasal transsphenoidal resection of craniopharyngioma and related tumors: a single-institution study. J Neurosurg. 124:627-638.2016; [PubMed: 26361276]

23. Komotar RJ, Starke RM, Raper DM, Anand VK, Schwartz TH. The endoscope-assisted ventral approach compared with open microscope-assisted surgery for clival chordomas. World Neurosurg. 76:318-327. 259-262.2011; [PubMed: 21986431]

24. Kong DS, Kim HY, Kim SH, Min JY, Nam DH, Park K, et al. Challenging reconstructive techniques for skull base defect following endoscopic endonasal approaches. Acta Neurochir (Wien). 153:807-813.2011; [PubMed: 21240667]

25. Koutourousiou M, Gardner PA, Fernandez-Miranda JC, Tyler-Kabara EC, Wang EW, Snyderman $\mathrm{CH}$. Endoscopic endonasal surgery for craniopharyngiomas: surgical outcome in 64 patients. $\mathrm{J}$ Neurosurg. 119:1194-1207.2013; [PubMed: 23909243]

26. Lee SJ, Lee D, Yoon TR, Kim HK, Jo HH, Park JS, et al. Surface modification of 3D-printed porous scaffolds via mussel-inspired polydopamine and effective immobilization of rhBMP-2 to promote osteogenic differentiation for bone tissue engineering. Acta Biomater. 40:182-191.2016; [PubMed: 26868173]

27. Leng LZ, Greenfield JP, Souweidane MM, Anand VK, Schwartz TH. Endoscopic, endonasal resection of craniopharyngiomas: analysis of outcome including extent of resection, cerebrospinal fluid leak, return to preoperative productivity, and body mass index. Neurosurgery. 70:110 124.2012; [PubMed: 21937943] 
28. Linsler S, Oertel J. Endoscopic endonasal transclival resection of a brainstem cavernoma: a detailed account of our technique and comparison with the literature. World Neurosurg. 84:20642071.2015; [PubMed: 26341427]

29. Luginbuhl AJ, Campbell PG, Evans J, Rosen M. Endoscopic repair of high-flow cranial base defects using a bilayer button. Laryngoscope. 120:876-880.2010; [PubMed: 20422679]

30. Mascarenhas L, Moshel YA, Bayad F, Szentirmai O, Salek AA, Leng LZ, et al. The transplanum transtuberculum approaches for suprasellar and sellar-suprasellar lesions: avoidance of cerebrospinal fluid leak and lessons learned. World Neurosurg. 82:186-195.2014; [PubMed: 23403355]

31. Nyberg E, Rindone A, Dorafshar A, Grayson WL. Comparison of 3D-printed poly- $\varepsilon$-caprolactone scaffolds functionalized with tricalcium phosphate, hydroxyapatite, Bio-Oss, or decellularized bone matrix. Tissue Eng Part A. 23:503-514.2017; [PubMed: 28027692]

32. Olson DR, Guiot G, Derome P. The symptomatic empty sella. Prevention and correction via the transsphenoidal approach. J Neurosurg. 37:533-537.1972; [PubMed: 5076370]

33. Rajappa P, Margetis K, Sigounas D, Anand V, Schwartz TH, Greenfield JP. Endoscopic endonasal transclival approach to a ventral pontine pediatric ependymoma. J Neurosurg Pediatr. 12:465468.2013; [PubMed: 23992235]

34. Randazzo M, Pisapia JM, Singh N, Thawani JP. 3D printing in neurosurgery: a systematic review. Surg Neurol Int. 7(Suppl 33):S801-S809.2016; [PubMed: 27920940]

35. Raza SM, Banu MA, Donaldson A, Patel KS, Anand VK, Schwartz TH. Sensitivity and specificity of intrathecal fluorescein and white light excitation for detecting intraoperative cerebrospinal fluid leak in endoscopic skull base surgery: a prospective study. J Neurosurg. 124:621-626.2016; [PubMed: 26295912]

36. Raza SM, Schwartz TH. Multi-layer reconstruction during endoscopic endonasal surgery: how much is necessary? World Neurosurg. 83:138-139.2015; [PubMed: 25009168]

37. Roy TD, Simon JL, Ricci JL, Rekow ED, Thompson VP, Parsons JR. Performance of degradable composite bone repair products made via three-dimensional fabrication techniques. J Biomed Mater Res A. 66:283-291.2003; [PubMed: 12888998]

38. Sigler AC, D’Anza B, Lobo BC, Woodard TD, Recinos PF, Sindwani R. Endoscopic skull base reconstruction: an evolution of materials and methods. Otolaryngol Clin North Am. 50:643653.2017; [PubMed: 28372814]

39. Tibbits S. 4D printing: multi-material shape change. Archit Design. 84:116-121.2014;

40. Vellutini EDA, Balsalobre L, Hermann DR, Stamm AC. The endoscopic endonasal approach for extradural and intradural clivus lesions. World Neurosurg. 82(6 Suppl):S106-S115.2014; [PubMed: 25496620]

41. Wannemuehler TJ, Rubel KE, Hendricks BK, Ting JY, Payner TD, Shah MV, et al. Outcomes in transcranial microsurgery versus extended endoscopic endonasal approach for primary resection of adult craniopharyngiomas. Neurosurg Focus. 41(6):E6.2016;

42. Yu AW, Khan M. On-demand three-dimensional printing of surgical supplies in conflict zones. J Trauma Acute Care Surg. 78:201-203.2015; [PubMed: 25539223]

43. Zwagerman NT, Zenonos G, Lieber S, Wang WH, Wang EW, Fernandez-Miranda JC, et al. Endoscopic transnasal skull base surgery: pushing the boundaries. J Neurooncol. 130:319330.2016; [PubMed: 27766473] 

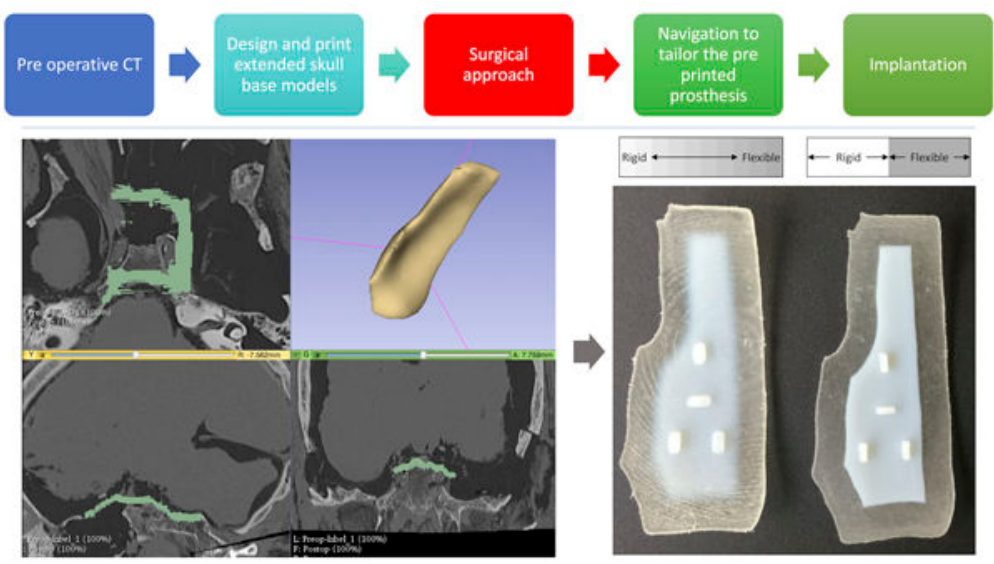

Fig. 1.

Extended skull base models: segmentation, printing, tailoring, and implantation steps. 


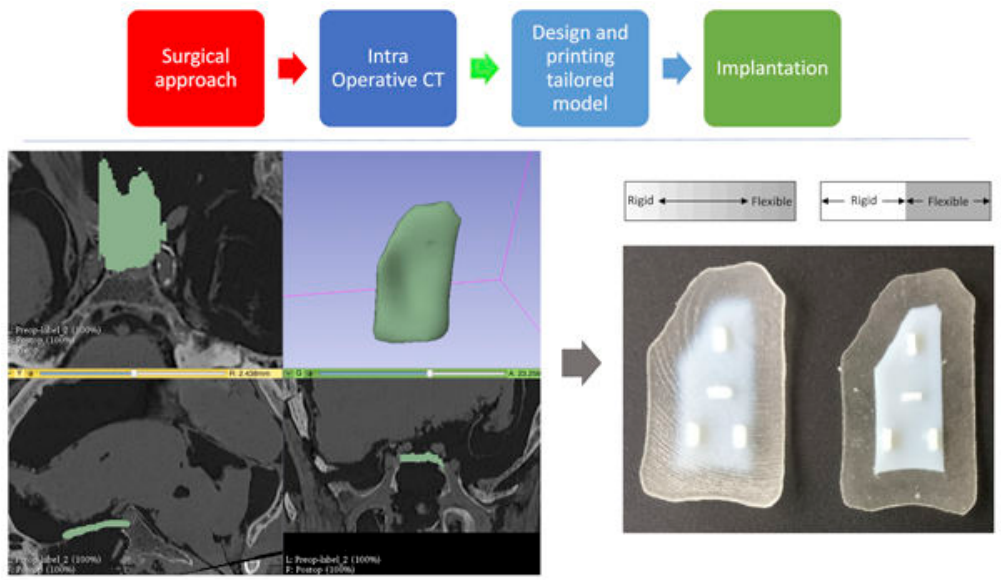

Fig. 2.

Tailored skull base models: segmentation, printing, and implantation steps. 


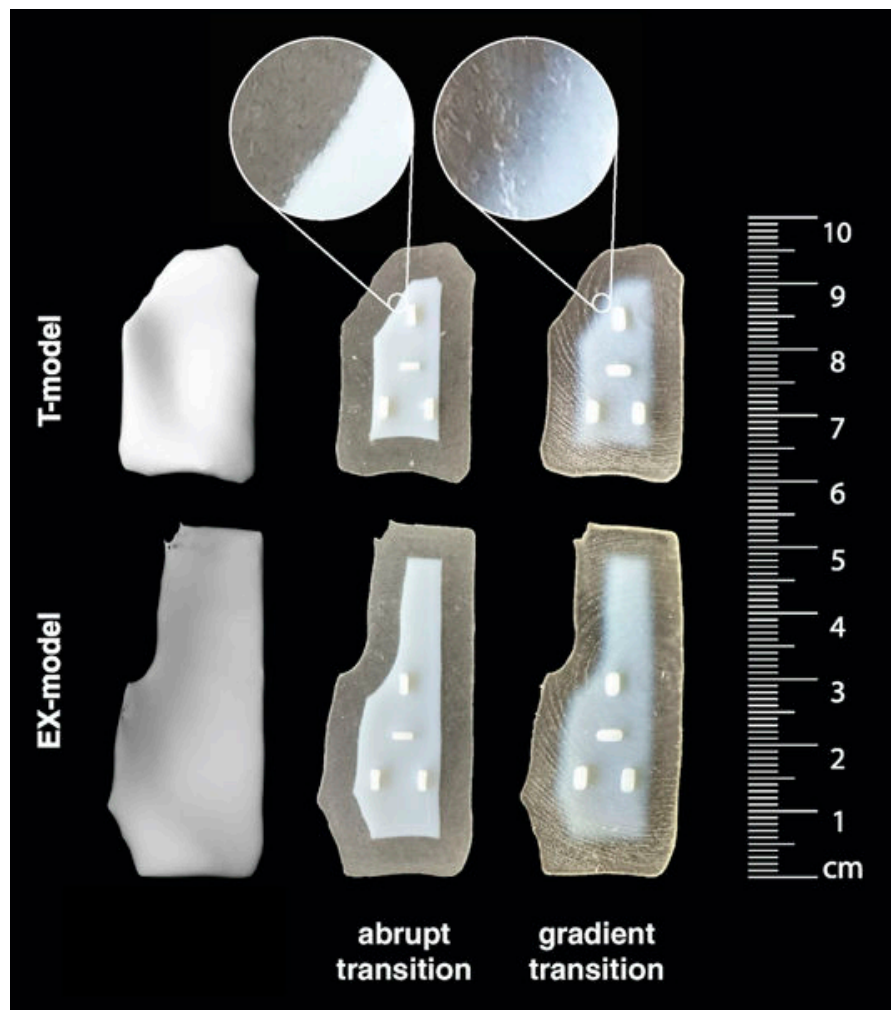

Fig. 3.

Extended skull base model (Ex-model) and tailored model (T-model), with abrupt and gradual center/edge material interface. 


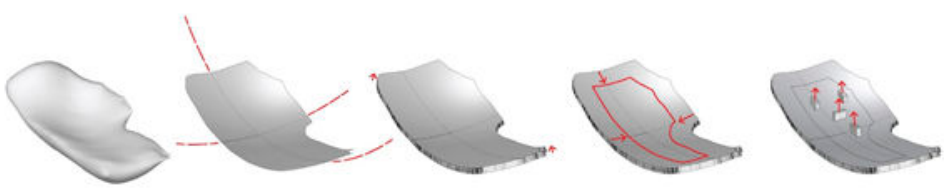

Fig. 4.

Prostheses modeling and postprocessing. Figure is available in color online only. 

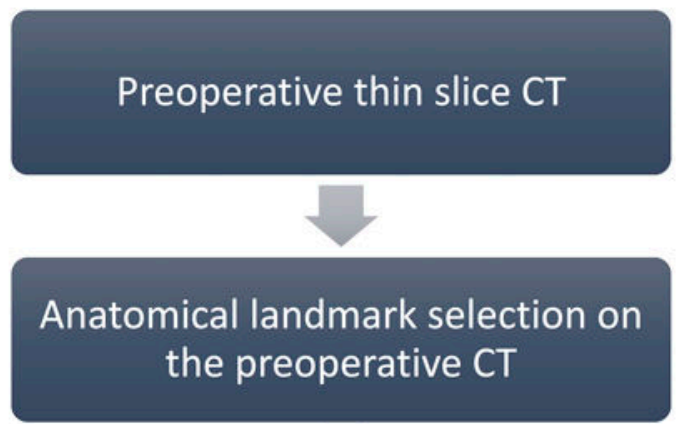

\section{Surgical approach}

Intraoperative validation of the preselected points as intraoperative landmarks

Mapping of the skull base defect edges

Restore registration using points on the prostheses corresponding to the intraoperative landmarks

Fig. 5.

Navigation transfer diagram. 


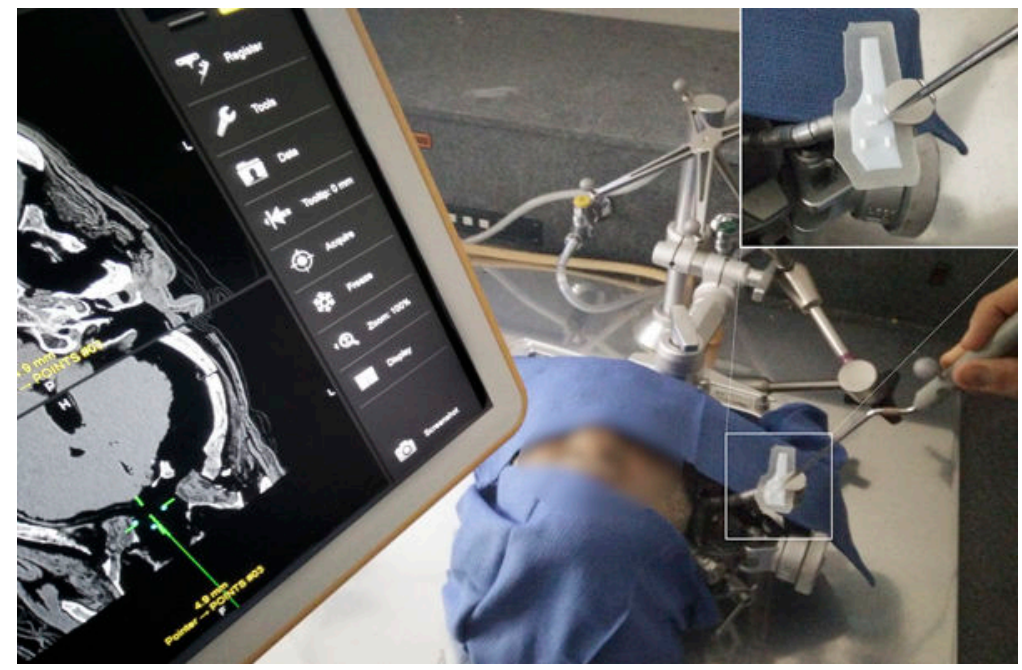

Fig. 6.

Photograph demonstrating navigation over the printed model. The inset shows a magnified view of the pointer being navigated over the model using the handles as landmarks. After transferring the registration to the Ex-model, it is possible to navigate on the prosthesis as if it was the patient skull base, allowing one to tailor the Ex-model to the defect. 


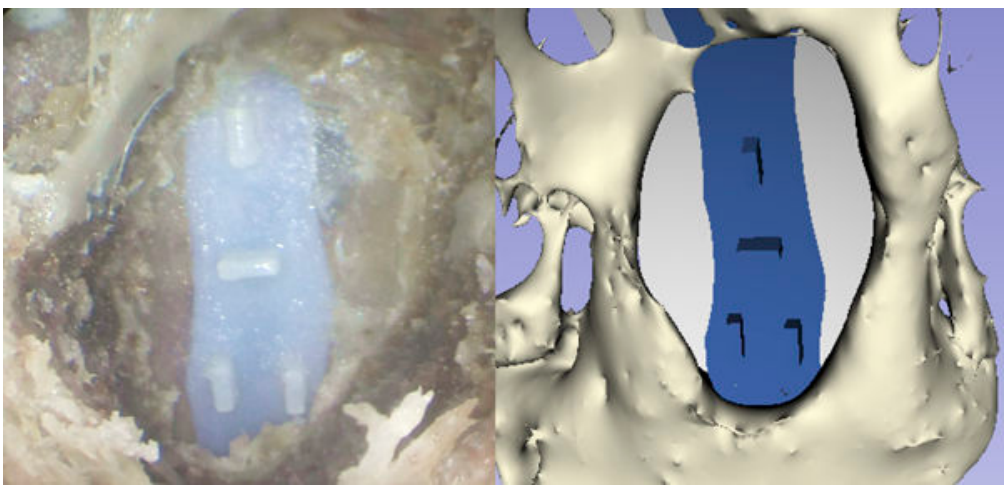

Fig. 7.

Ex-model cadaver implantation and 3D rendering (corresponds to Video 1). 